\title{
Neurological Manifestations of Acute Posterior Multifocal Placoid Pigment Epitheliopathy
}

\author{
Hussein Algahtani $^{a}$ \\ Ashjan Alkhotani $^{\mathrm{a}}$ \\ Bader Shirah ${ }^{b}$ \\ aKing Abdulaziz Medical City/ \\ King Saud bin Abdulaziz University \\ for Health Sciences, Jeddah, \\ Saudi Arabia \\ ${ }^{\mathrm{b}}$ King Abdullah International \\ Medical Research Center/ \\ King Saud bin Abdulaziz University \\ for Health Sciences, Jeddah, \\ Saudi Arabia
}

\begin{abstract}
Background and Purpose Acute posterior multifocal placoid pigment epitheliopathy (APMPPE) is an immune-mediated chorioretinal disease that causes acute visual symptoms with characteristic ophthalmoscopic findings. Neurological complications are rarely reported in the literature. Here we report two new cases of APMPPE that presented with neurological manifestations, one of which was associated with peripheral neuropathy, which has not been described before.
\end{abstract}

Methods A retrospective database review of all patients with a diagnosis of APMPPE was performed. Clinical, ophthalmological, and neurological data were analyzed, and only cases of APMPPE with neurological complications were included. A literature review of several databases was also performed, and previous case reports were reviewed and analyzed in detail.

Results In total, 56 cases of APMPPE-associated neurological complications were included in the analyses: 54 from the literature and 2 from our own practice. The most common complication was cerebral vasculitis, which affected 28 patients (50\%), followed by headaches in 15 patients $(26.8 \%)$. The other complications include sixth-cranial-nerve palsy, transient hearing loss, meningoencephalitis, cavernous sinus thrombosis, and viral meningitis.

Conclusions This report adds to the literature of a novel association of APMPPE with peripheral neuropathy, and comprehensively reviews the neurological manifestations of this disease. A high level of suspicion should be applied when dealing with a case of APMPPE. We recommend applying detailed clinical neurological examinations and magnetic resonance imaging to APMPPE patients, and then early steroid treatment if the examination is positive or even suspicious. Early treatment with steroids and long-term treatment with immunosuppressive azathioprine with interval neurological evaluations will contribute positively to the outcomes and avoid fatal complications, namely strokes.

Key Words acute posterior multifocal placoid pigment epitheliopathy, neurological complications, peripheral neuropathy, corticosteroids.
Received February 2, 2016

Revised April 4, 2016

Accepted April 5, 2016

\section{Correspondence}

Hussein Algahtani, MD, FRCPC

King Abdulaziz Medical City/

King Saud bin Abdulaziz University

for Health Sciences, Jeddah 21483,

Saudi Arabia

Tel 966-556633130

E-mail halgahtani@hotmail.com

\section{INTRODUCTION}

Acute posterior multifocal placoid pigment epitheliopathy (APMPPE) is a rare immunemediated chorioretinal disease that affects young adults. It typically affects both eyes, and patients usually present with binocular visual blurring, metamorphopsia, or scotomas with characteristic fundus findings. ${ }^{1}$ Although this is a disease of the eye, neurological and systemic manifestations can also occur. It has been associated with multiple complications in the central nervous system (CNS), including cerebral vasculitis, headaches, aseptic meningitis, meningoencephalitis, sixth-cranial-nerve palsy, transient hearing loss, and cavernous sinus thrombosis. ${ }^{2}$

This study adds two new cases of APMPPE with CNS manifestations to the literature, and

(®) This is an Open Access article distributed under the terms of the Creative Commons Attribution Non-Commercial License (http://creativecommons.org/licenses/by-nc/3.0) which permits unrestricted non-commercial use, distribution, and reproduction in any medium, provided the original work is properly cited. 
we describe a novel association with peripheral neuropathy that has not been reported before. In addition, we review the literature and previous case reports, and discuss the diseases in detail, including important steps in their diagnosis and management.

\section{METHODS}

A retrospective observational study was performed at the neurology unit in King Abdulaziz Medical City, Jeddah, Saudi Arabia. We reviewed the charts of all patients who presented with retinal disease between January 2000 and December 2015, and selected only those with a diagnosis of APMPPE with neurological complications. The variables collected were age, sex, comorbidities, and clinical, ophthalmological, and neurological data, investigations, treatments, and outcomes. We also reviewed the literature in Medline, Ovid, EMBASE, ProQuest, Google Scholar, and PubMed databases for cases of APMPPE-associated neurological complications, including cerebrovascular disease. This study was approved by the institutional review board of King Abdullah International Medical Research Center, and consent was waived in accordance with the institutional policy since this was an observational study (IRB approval number: RYD16-417780-56087). Patient confidentiality was assured by identifying cases using only file numbers without names or photographs.

\section{Case report 1}

A previously healthy 26 -year-old right-handed woman developed an influenza-like illness with myalgia, arthralgia, and cough. One week later she experienced flashing lights, floaters, blurred vision, and sore eyes, and nonspecific holocephalic headache. Her symptoms improved over a period of 3 weeks. Two months later she developed imbalance, rightsided numbness, and weakness. There was no past history of any medical illness, drug or alcohol abuse, smoking, or recent international travel.

A general examination produced normal findings, including for vital signs. In a neurological examination her visual acuity was 20/30 in the right eye and 20/40 in the left eye, with both discs demonstrating moderate nasal, superior, and inferior swelling. Multiple creamy yellow patches involving the retinal pigment epithelium (RPE) were seen in both posterior poles (Fig. 1). The visual fields as tested using Goldman perimetry were normal except for enlargement of the blind spots. Extraocular eye movements were full, and her pupils were equally reactive to light with no relative afferent pupillary defect. The other cranial nerves were normal. A motor examination showed right-sided weakness ( 3 out of 5 ), a pyramidal pattern, with pronator drift and spastic catch. The sensory examination revealed impaired primary sensory modalities on the right side especially in the light touch and pinprick sensations. Cerebellar functions and coordination were normal. The findings of basic laboratory and X-ray investigations were normal including a vasculitis screen and serologies for all related organisms. A cerebrospinal fluid (CSF) analysis showed 10 white blood cells, 94\% lymphocytes, no red blood cells, and normal protein and glucose levels. Culture and viral studies using the polymerase chain reaction for most viruses produced negative results. Computed tomography (CT) and magnetic resonance imaging (MRI) showed infarcts at the anterior and posterior limbs of the left internal capsule extending into the putamen, left corona radiata, right pericallosal white matter, and high right frontal centrum semiovale (Fig. 2). Conventional angiography showed multifocal narrowing of branches of the anterior and middle cerebral arteries bilaterally.

The patient was started on intravenous pulse steroid therapy followed by oral prednisolone at $60 \mathrm{mg}$ once daily in a gradually decreasing dose and aspirin at $81 \mathrm{mg}$ once daily. Her symptoms improved, and the findings of neurological
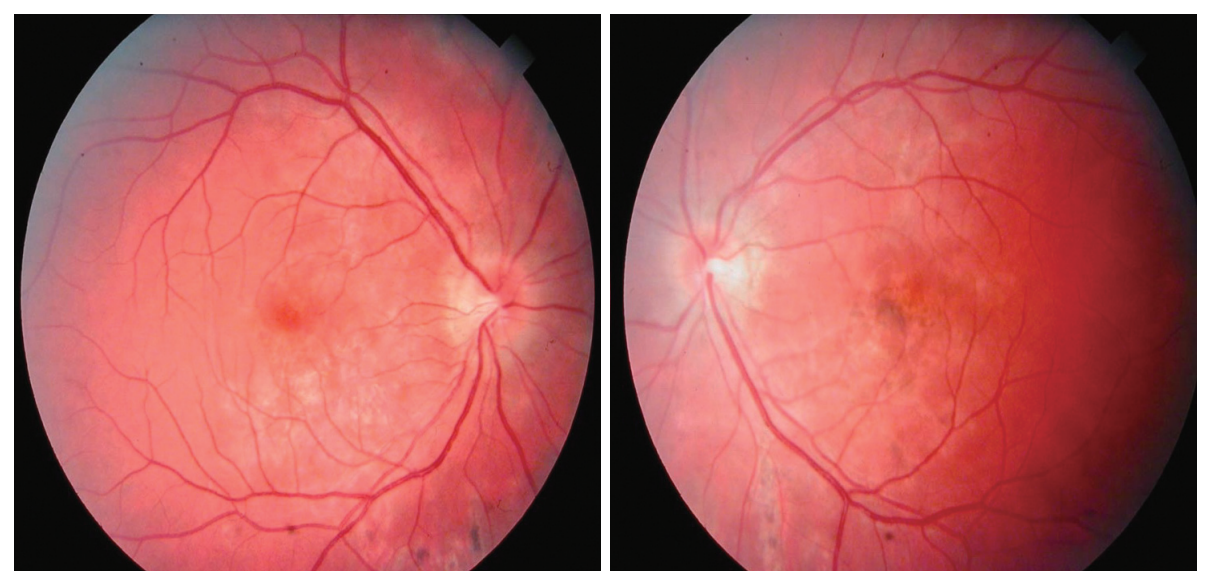

Fig. 1. Both posterior poles of the first case showing multiple creamy yellow patches involving the retinal pigment epithelium. 

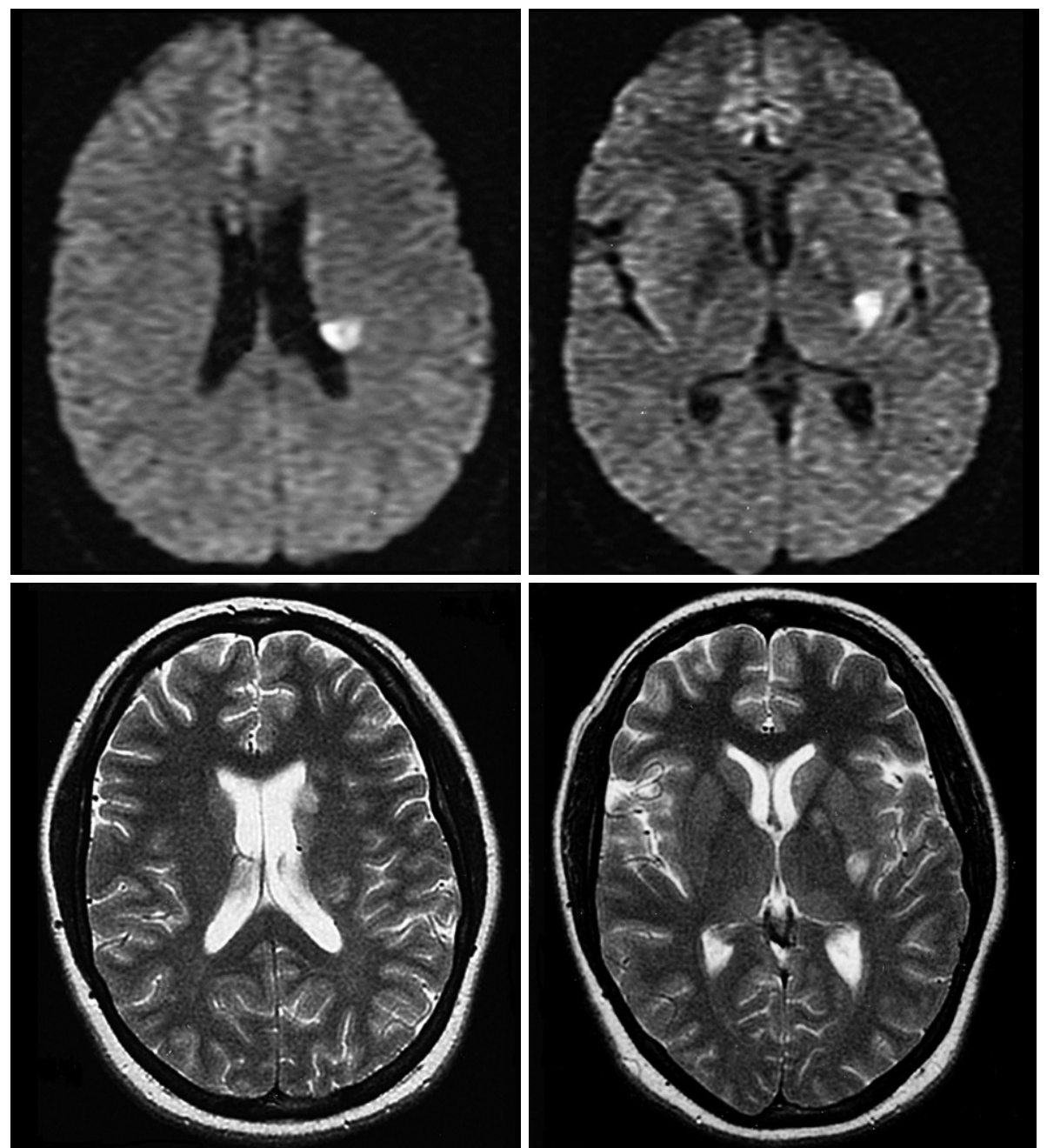

Fig. 2. MRI of the brain showing infarcts at the posterior limb of the left internal capsule extending into the putamen, corona radiata, pericallosal white matter, and posterior frontal centrum semiovale.

and eye examinations were normal in a follow-up clinic visit 2 months later.

\section{Case report 2}

A 49-year-old man developed a subacute, intense, bifrontal headache associated with blurring of vision. He had no history of diabetes, hypertension, or alcohol intake. Three weeks later he developed left frontal and right temporal infarctions, as confirmed by MRI. His retina showed characteristic changes of APMPPE with no emboli evident. Fluorescence angiography and other ophthalmological tests revealed typical changes of APMPPE (Fig. 3). The patient was diagnosed as APMPPE based on the ophthalmological examination, and he was treated with intravenous pulse steroid therapy followed by oral steroids. Cerebral angiography confirmed the presence of vasculitis. The findings of transesophageal echocardiography and 48-hour Holter monitoring were normal. Laboratory investigations showed a normal erythrocyte sedi- mentation rate (ESR), C-reactive protein, creatinine level, and liver enzymes. Additional tests revealed normal serum levels of gammaglobulins, antinuclear antibodies, and complement. Antineutrophil cytoplasmic antibodies, rheumatoid factor, antiphospholipid and anticardiolipin antibodies, as well as serologies for borreliosis, syphilis, human immunodeficiency virus, and hepatitis $B$ and $C$ were all negative.

The condition of the patient improved after administering high-dose steroids and clopidogrel at $75 \mathrm{mg}$ once daily. Prednisone was gradually tapered off over a 6-week period. He was left with a visual acuity of 20/100 in both eyes and left superior homonymous quadrantanopia. Eight months later he developed bilateral leg numbness and tingling. The findings of electrophysiological studies were consistent with axon-length-dependent polyneuropathy that preferentially affected the motor fibers. The findings of MRI of the whole spine were normal, and repeated MRI of the brain showed resolution of the previous infarctions with no new lesions. 

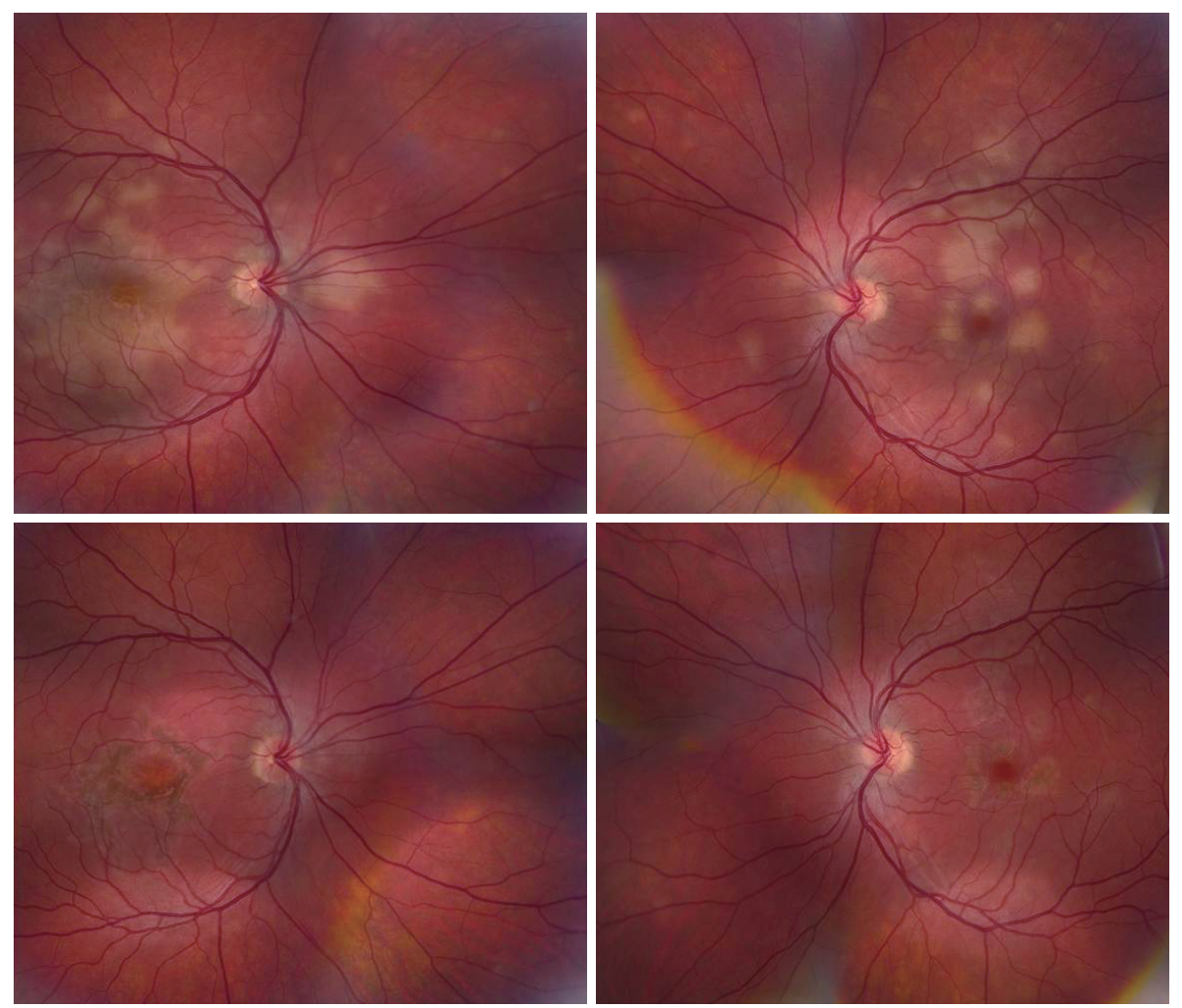

Fig. 3. Fundus photography of the second case showing multiple creamy yellow patches involving the retinal pigment epithelium before and after acute posterior multifocal placoid pigment epitheliopathy treatment.

Gabapentin was started at $300 \mathrm{mg}$ three times daily, which improved the sensory symptoms. The patient experienced no further neurological events. Additional investigations including of the B12 level, glucose tolerance test, celiac-disease workup, connective-tissue disease, and serum protein electrophoresis did not reveal any cause of his polyneuropathy. The patient unfortunately refused to receive a second course of steroids or any immunosuppressive therapy. Two months later he was seen in ophthalmology and neurology clinics, at which time both his visual and neurological functions had improved.

\section{RESULTS}

Our literature review revealed 54 well-documented cases of APMPPE-associated neurological complications. A review of our own data identified 105 different retinal disorders. Only two cases of APMPPE were identified, both of which presented with the classical ocular manifestations of APMPPE with the first presentation being to a neurology clinic. Adding our 2 patients to the cases already in the literature brought the total number of cases in our study to 56 . Thirty-seven were male (66.1\%) and 19 were female (33.9\%), giving a male-to-female ratio 1.95:1, and they were aged $28.9 \pm 11.0$ years (mean $\pm \mathrm{SD}$, range $=15-58$ years). The most common complication was cerebral vasculitis, which affected $28 \mathrm{pa}$ tients (50\%), followed by headaches in 15 patients (26.8\%) (Table 1). The other complications included sixth-cranialnerve palsy, transient hearing loss, meningoencephalitis, cavernous sinus thrombosis, and viral meningitis. Forty patients $(71.4 \%)$ recovered completely, while ten $(17.9 \%)$ had long-term disability. Six patients (10.7\%) died, all of whom were in the cerebral vasculitis group. The main cause of death was stroke-related complications including status epilepticus, cerebral edema, and uncal herniation. The CSF was analyzed in 36 patients (64.3\%), with abnormal results in $28(77.8 \%)$, including pleocytosis (mononuclear) and elevated protein. Headaches were reported in 31 patients (55.4\%), and this was the only neurological manifestation in 15 (48.4\%) of them. A detailed description of the headache in terms of its type, location, frequency, and severity was lacking for most of the studies reviewed.

Cerebral angiography was performed in 18 patients (32.1\%), CT in 9 (16.1\%), and MRI in $45(80.4 \%)$. MRI was the most sensitive modality of investigation for detecting neurological complications of APMPPE. The findings of cerebral angiographies were all abnormal except for one in which the vessel images showed evidence of vasculitic changes. Steroids has been used by 50 patients (89.3\%), and long-term immunosuppressive therapy was given to $10(17.9 \%)$. The 


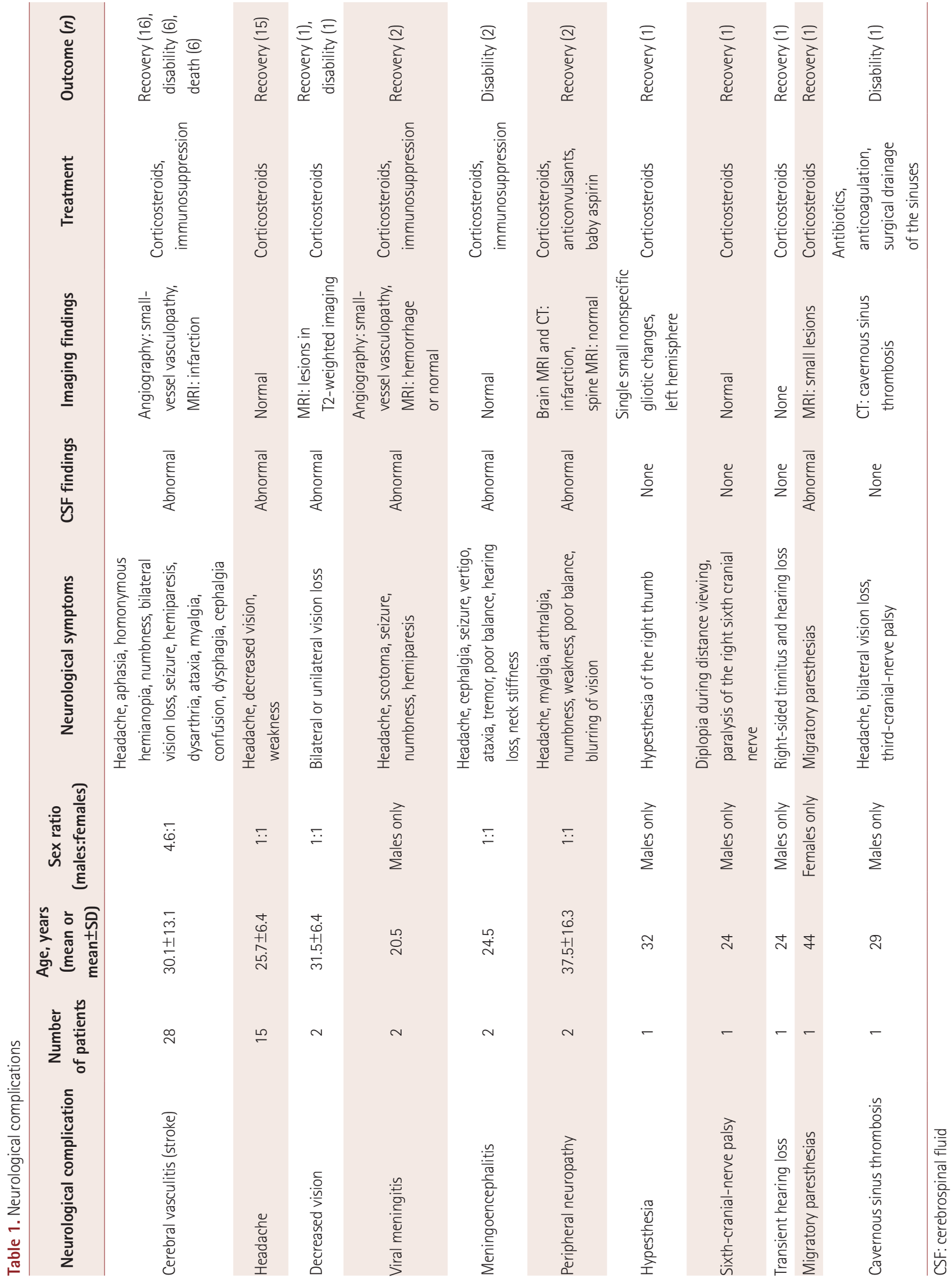


long-term immunosuppressives used included azathioprine in 6 patients (10.7\%), cyclophosphamide in $3(5.4 \%)$, and mitoxantrone in $1(1.8 \%)$. The time from the onset of ocular symptoms to the onset of CNS symptoms was $50.1 \pm 110.6$ days (range $=0-336$ days). One patient experienced the onset of the neurological complication at 6 years after the ocular disease, and we considered this patient to be an outlier that was attributable to either treatment failure or premature discontinuation of immunosuppressive therapy. Histopathological studies had been reported previously for three autopsies that demonstrated granulomatous vasculitis of the small and medium cerebral vessels, with multinucleated giant-cell deposition and fibrinoid necrosis. Furthermore, generalized granulomas were found in several organs such as the lung parenchyma, lymph nodes, heart, liver, and spleen.

\section{DISCUSSION}

In 1968, Gass ${ }^{3}$ described clinical and angiographic findings in three young women who presented with a short history of painless loss of central vision associated with multiple cream-colored lesions with ill-defined margins concentrated in the posterior pole, and apparently deep in the retina. These placoid lesions at the level of the RPE and choroid resolved spontaneously over a few weeks, leaving scarring but with good recovery of visual functions - the condition was therefore named APMPPE. A systemic investigation did not reveal a cause for the ocular disease. ${ }^{3}$ Although the clinical findings of this disease may have been reported earlier, Gass ${ }^{3}$ was the first to link the clinical and angiographic findings with a proposed pathogenesis. Further reports soon followed, and a picture developed of a rare, acute, selflimiting inflammatory disorder with a typical clinical pattern sometimes with profound loss of vision, but usually with remarkable visual recovery (if the fovea is spared) despite substantial residual scarring of the RPE., ${ }^{4,5}$ APMPPE is characterized by the sudden appearance of multiple posterior pole lesions that are yellow-to-white in color. Patients usually present with binocular visual blurring, metamorphopsia, or scotomas with characteristic fundus findings. ${ }^{6}$

The rarity of APMPPE means that accurate estimates of its incidence and prevalence are not available. There are few systematic reports in the literature, and only case reports and series have been published. Most of the studies have been retrospective, as is often the case with rare diseases. The disease is seen mostly in young patients, at a mean age of around 25 years (range $=7-51$ years). It has an equal sex distribution, and affects both eyes in $>85 \%$ of cases. ${ }^{7}$ However, our results indicate that the neurological complications occur in males almost twice as often as in females. This observa- tion is supported by the excellent literature review of Luneau et al. ${ }^{8}$ of APMPPE cases associated with cerebrovascular complications that have been published in the French and English literature.

The exact etiology of APMPPE is unknown, and there are at least two possible mechanisms explaining this condition. The disease was thought to be an inflammatory process that begins either at the level of the RPE, as Gass ${ }^{3}$ mentioned, or at the level of small choroidal arterioles with vasculitis and ischemic changes. ${ }^{9}$ The associated systemic vasculitis provides support for the choroid as being primarily involved via a diffuse vasculitic process that interrupts choroidal perfusion and causes the characteristic fundus findings in APMPPE. Choroidal ischemia leads to a disturbance of the RPE barrier. ${ }^{9}$ Another proposed mechanism for APMPPE is a choroidal delayed-type hypersensitivity reaction with activation of sensitized T lymphocytes to an unknown agent resulting in choroidal occlusive vasculitis. It has been reported to be associated with numerous infections and diseases that are caused by delayed-type hypersensitivity reactions such as sarcoidosis, pulmonary tuberculosis, schistosomiasis, ulcerative colitis, and acute group A streptococcal infection. ${ }^{10,11}$ About $40 \%$ of patients diagnosed with APMPPE report influenza-like symptoms prior to the onset of the visual symptoms. ${ }^{12,13}$ APMPPE has also been reported following vaccinations against meningococcal $C$, mumps, influenza, or hepatitis $\mathrm{B}$ viruses, suggesting an immune-mediated mechanism rather than a direct effect of the infectious agent. . $^{14,15}$ In addition, certain human leukocyte antigen (HLA) haplotypes have been shown to be associated with the disorder, with $56.7 \%$ of patients with APMPPE reported to be positive for HLA-DR2, while $40 \%$ express HLA-B7. These major histocompatibility complex proteins may present viral or bacterial antigens to helper and cytotoxic T cells, and activate the immune response leading to capillary and pigment epithelial cell inflammation. ${ }^{16}$

Systemic complications of APMPPE have been described previously. These may involve the skin (erythema nodosum), kidneys (nephritis with urine casts), muscles, thyroid glands (thyroiditis), vasculitis of systemic blood vessels, and a high ESR. ${ }^{17}$ Based on cerebral angiographies, Reichhart ${ }^{18}$ suggested that the disease represents a "uveocerebral" vasculitic syndrome. The differential diagnosis of this syndrome includes Vogt-Koyanagi-Harada disease, sarcoidosis, Behçet disease, systemic lupus erythematosus, and Crohn's disease. ${ }^{19} \mathrm{~A}$ blood workup to exclude systemic vasculitis includes ESR, C-reactive protein, C-anti-neutrophil cytoplasmic antibody, rheumatoid factor, antinuclear antibody, and anti-DNA. ${ }^{17}$

APMPPE was first described in 1968, and the CNS involvement was described first by Holt et al. ${ }^{5}$ in 1976 . Those 
authors described a 22-year-old male with attacks of rightsided weakness and aphasia followed by left-sided numbness most likely representing transient ischemic attacks. The cerebral angiogram was consistent with cerebral vasculitis. ${ }^{5}$ Several neurological and systemic manifestations have been reported subsequently, with the CNS complications including cerebral vasculitis, headaches, aseptic meningitis, meningoencephalitis, sixth-cranial-nerve palsy, transient hearing loss, and cavernous sinus thrombosis. ${ }^{19-25}$ The present report not only includes a rare complication of a rare disease, but also an association with polyneuropathy that has not been reported previously. Whether the polyneuropathy is a sequela, coincidence, or an association remains to be clarified.

Since APMPPE is generally self-limiting, there is no rationale for treatment if no neurological complication is encountered. The outcome for the visual system without treatment is characteristically good, and a gradual improvement in visual acuity occurs over several months, with most eyes achieving a visual acuity of 20/30 or better. ${ }^{26}$ Steroid therapy may be indicated for extensive disease that involves the fovea. Steroids may offer a theoretical advantage in shortening the disease course or modifying its effects on central vision. ${ }^{27,28}$

The scenario is different in patients with APMPPE and neurological symptoms or complications, with aggressive management involving steroids and immunosuppressive medications being strongly indicated. Weinstein et al. ${ }^{29}$ suggested that once cerebral vasculitis has been diagnosed, it should be treated with steroid for 4 months in order to avoid stroke occurrence. Stoll et al. ${ }^{30}$ applied steroids for 2 months followed by azathioprine for long-term immunosuppression. Comu et al. ${ }^{31}$ suggested stopping immunosuppressive therapy after 6-12 months. Based on our literature review, we recommend using steroids for 2-3 months and immunosuppressive therapy (azathioprine) for 12 months, during which full ophthalmological and neurological evaluations should be performed at 3-month intervals.

The prognosis of the neurological complications varies, with most people either recovering completely or stabilizing with no further deterioration. Factors that are prognostic of a poor outcome include the occurrence of stroke, lack of spontaneous remission with protracted disease course, and rapid tapering of immunosuppressive therapy. ${ }^{19,32,33}$

APMPPE is a well-established disease with classical clinical presentation and prognosis, but extraocular manifestations including neurological complications are quite rare. Cerebral vasculitis and strokes are the most commonly reported neurological complications, affecting half of the reported cases. This report adds to the literature by describing a novel association of APMPPE with peripheral neuropathy as well as comprehensively reviewing the neurological manifestations of the disease. A high level of suspicion should be applied when dealing with a case of APMPPE. We recommend applying detailed clinical neurological examinations and MRI to APMPPE patients, and then early steroid treatment if the examination is positive or even suspicious. Early treatment with steroids and long-term treatment with immunosuppressive azathioprine with interval neurological evaluations will contribute positively to the outcomes and avoid fatal complications, namely strokes.

\section{Conflicts of Interest}

The authors have no financial conflicts of interest.

\section{REFERENCES}

1. Vianna RN, Socci D, Nehemy MB, Deschênes J, Burnier MN Jr. The white dot syndromes. Arq Bras Oftalmol 2007;70:554-562.

2. Case D, Seinfeld J, Kumpe D, Folzenlogen Z, Jones W, Simpson J, et al. Acute posterior multifocal placoid pigment epitheliopathy associated with stroke: a case report and review of the literature. J Stroke Cerebrovasc Dis 2015;24:e295-e302.

3. Gass JD. Acute posterior multifocal placoid pigment epitheliopathy. Arch Ophthalmol 1968;80:177-185.

4. Deutman AF, Oosterhuis JA, Boen-Tan TN, Aan de Kerk AL. Acute posterior multifocal placoid pigment epitheliopathy. Pigment epitheliopathy of choriocapillaritis? Br J Ophthalmol 1972;56:863-874.

5. Holt WS, Regan CD, Trempe C. Acute posterior multifocal placoid pigment epitheliopathy. Am J Ophthalmol 1976;81:403-412.

6. Oleszczuk JD, Saeed MU. Neurological symptoms associated with acute multifocal placoid pigment epitheliopathy: treatment dilemma and diagnostic issues. Semin Ophthalmol 2015;30:238-240.

7. Roberts TV, Mitchell P. Acute posterior multifocal placoid pigment epitheliopathy: a long-term study. Aust N Z J Ophthalmol 1997;25:277281.

8. Luneau K, Newman NJ, Srivastava S, Biousse V. A case of acute posterior multifocal placoid pigment epitheliopathy with recurrent stroke. J Neuroophthalmol 2009;29:111-118.

9. Bouchenaki N, Cimino L, Auer C, Tao Tran V, Herbort CP. Assessment and classification of choroidal vasculitis in posterior uveitis using indocyanine green angiography. Klin Monbl Augenheilkd 2002;219:243-249.

10. Di Crecchio L, Parodi MB, Saviano S, Ravalico G. Acute posterior multifocal placoid pigment epitheliopathy and ulcerative colitis: a possible association. Acta Ophthalmol Scand 2001;79:319-321.

11. Darugar A, Mathian A, Lehoang P, Bodaghi B. Acute posterior multifocal placoid pigment epitheliopathy as the initial manifestation of sarcoidosis. J Ophthalmic Vis Res 2011;6:338-343.

12. Thomson SP, Roxburgh ST. Acute posterior multifocal placoid pigment epitheliopathy associated with adenovirus infection. Eye (Lond) 2003;17:542-544.

13. Azar P Jr, Gohd RS, Waltman D, Gitter KA. Acute posterior multifocal placoid pigment epitheliopathy associated with an adenovirus type 5 infection. Am J Ophthalmol 1975;80:1003-1005.

14. Brézin AP, Massin-Korobelnik P, Boudin M, Gaudric A, LeHoang P. Acute posterior multifocal placoid pigment epitheliopathy after hepatitis B vaccine. Arch Ophthalmol 1995;113:297-300.

15. Fine HF, Kim E, Flynn TE, Gomes NL, Chang S. Acute posterior multifocal placoid pigment epitheliopathy following varicella vaccination. Br J Ophthalmol 2010;94:282-283, 363.

16. Wolf MD, Folk JC, Panknen CA, Goeken NE. HLA-B7 and HLADR2 antigens and acute posterior multifocal placoid pigment epitheliopathy. Arch Ophthalmol 1990;108:698-700. 
17. Hsu CT, Harlan JB, Goldberg MF, Dunn JP. Acute posterior multifocal placoid pigment epitheliopathy associated with a systemic necrotizing vasculitis. Retina 2003;23:64-68.

18. Reichhart M. Acute posterior multifocal placoid pigment epitheliopathy (APMPPE). In: Caplan LR, Bogousslavsky J, editors. Uncommon causes of stroke. 2nd ed. Cambridge: Cambridge University Press, 2008;237-246.

19. Tsang BK, Chauhan DS, Haward R, Whiteman I, Frayne J, McLean C. Fatal ischemic stroke complicating acute multifocal placoid pigment epitheliopathy: histopathological findings. J Neuroophthalmol 2014;34:10-15.

20. Clearkin LG, Hung SO. Acute posterior multifocal placoid pigment epitheliopathy associated with transient hearing loss. Trans Ophthalmol Soc U K 1983;103(Pt 5):562-564.

21. Kersten DH, Lessell S, Carlow TJ. Acute posterior multifocal placoid pigment epitheliopathy and late-onset meningo-encephalitis. Ophthalmology 1987;94:393-396.

22. Kline DC, Vitale A, Warner JE. Acute multifocal placoid pigment epitheliopathy associated with cavernous sinus thrombosis. Ocul Immunol Inflamm 2007;15:443-446.

23. Gibelalde A, Bidaguren A, Ostolaza JI, Cortázar L, Irigoyen C. [Pigmentary epitheliopathy multifocal acute placoid associated with paralysis of VI cranial par]. Arch Soc Esp Oftalmol 2009;84:159-162.

24. Thomas BC, Jacobi C, Korporal M, Becker MD, Wildemann B, Mackensen F. Ocular outcome and frequency of neurological manifestations in patients with acute posterior multifocal placoid pigment epitheliopathy (APMPPE). J Ophthalmic Inflamm Infect 2012;2:125-131.

25. O'Halloran HS, Berger JR, Lee WB, Robertson DM, Giovannini JA, Krohel GB, et al. Acute multifocal placoid pigment epitheliopathy and central nervous system involvement: nine new cases and a review of the literature. Ophthalmology 2001;108:861-868.

26. Vianna R, van Egmond J, Priem H, Kestelyn P. Natural history and visual outcome in patients with APMPPE. Bull Soc Belge Ophtalmol 1993;248:73-76.

27. Williams DF, Mieler WF. Long-term follow-up of acute multifocal posterior placoid pigment epitheliopathy. Br J Ophthalmol 1989;73:985-990.

28. Bridges WJ, Saadeh C, Gerald R. Acute posterior multifocal placoid pigment epitheliopathy in a patient with systemic-onset juvenile rheumatoid arthritis: treatment with cyclosporin A and prednisone. Arthritis Rheum 1995;38:446-447.

29. Weinstein JM, Bresnick GH, Bell CL, Roschmann RA, Brooks BR, Strother CM. Acute posterior multifocal placoid pigment epitheliopathy associated with cerebral vasculitis. J Clin Neuroophthalmol 1988; 8:195-201.

30. Stoll G, Reiners K, Schwartz A, Kaup FG, Althaus C, Freund HJ. Acute posterior multifocal placoid pigment epitheliopathy with cerebral involvement. J Neurol Neurosurg Psychiatry 1991;54:77-79.

31. Comu S, Verstraeten T, Rinkoff JS, Busis NA. Neurological manifestations of acute posterior multifocal placoid pigment epitheliopathy. Stroke 1996;27:996-1001.

32. Wilson CA, Choromokos EA, Sheppard R. Acute posterior multifocal placoid pigment epitheliopathy and cerebral vasculitis. Arch Ophthalmol 1988;106:796-800.

33. de Vries JJ, den Dunnen WF, Timmerman EA, Kruithof IG, De Keyser J. Acute posterior multifocal placoid pigment epitheliopathy with cerebral vasculitis: a multisystem granulomatous disease. Arch Ophthalmol 2006;124:910-913. 